\title{
SOME MARTINGALES ASSOCIATED WITH MULTIVARIATE BESSEL PROCESSES
}

\author{
MIKLOS KORNYIK, MICHAEL VOIT, AND JEANNETTE H.C. WOERNER
}

\begin{abstract}
We study Bessel processes on Weyl chambers of types A and B on $\mathbb{R}^{N}$. Using elementary symmetric functions, we present several space-timeharmonic functions and thus martingales for these processes $\left(X_{t}\right)_{t \geq 0}$ which are independent from one parameter of these processes. As a consequence, $p(y):=\mathbb{E}\left(\prod_{i=1}^{N}\left(y-X_{t}^{i}\right)\right)$ can be expressed via classical orthogonal polynomials. Such formulas on characteristic polynomials admit interpretations in random matrix theory where they are partially known by Diaconis, Forrester, and Gamburd.
\end{abstract}

\section{INTRODUCTION}

Interacting Calogero-Moser-Sutherland particle models on $\mathbb{R}$ with $N$ particles can be described via Bessel processes $\left(X_{t}\right)_{t \geq 0}$ associated with root systems; see e.g. CGY, GY, R, RV1, RV2, DV, AKM1, AKM2, These processes are classified via root systems and finitely many multiplicity parameters which control the interaction. In this paper, we only study the root systems $A_{N-1}$ and $B_{N}$.

In the case $A_{N-1}$, we have a multiplicity $k \in\left[0, \infty\left[,\left(X_{t}\right)_{t \geq 0}\right.\right.$ lives on the closed Weyl chamber

$$
C_{N}^{A}:=\left\{x \in \mathbb{R}^{N}: \quad x_{1} \geq x_{2} \geq \ldots \geq x_{N}\right\}
$$

the generator of the transition semigroup is

$$
\Delta_{k} f:=\frac{1}{2} \Delta f+k \sum_{i=1}^{N}\left(\sum_{j: j \neq i} \frac{1}{x_{i}-x_{j}}\right) \frac{\partial}{\partial x_{i}} f,
$$

and we assume reflecting boundaries, i.e., the domain of $\Delta_{k}$ may be chosen as $D\left(\Delta_{k}\right):=\left\{\left.f\right|_{C_{N}^{A}}: f \in C^{(2)}\left(\mathbb{R}^{N}\right), f\right.$ invariant under all coordinate-premutations $\}$.

In the case $B_{N}$, we have two multiplicities $k_{1}, k_{2} \geq 0$, the processes live on

$$
C_{N}^{B}:=\left\{x \in \mathbb{R}^{N}: \quad x_{1} \geq x_{2} \geq \ldots \geq x_{N} \geq 0\right\},
$$

the generator of the transition semigroup is

$$
\Delta_{k_{1}, k_{2}} f:=\frac{1}{2} \Delta f+k_{2} \sum_{i=1}^{N} \sum_{j: j \neq i}\left(\frac{1}{x_{i}-x_{j}}+\frac{1}{x_{i}+x_{j}}\right) \frac{\partial}{\partial x_{i}} f+k_{1} \sum_{i=1}^{N} \frac{1}{x_{i}} \frac{\partial}{\partial x_{i}} f,
$$

and we again assume reflecting boundaries.

Date: August 30, 2019.

2010 Mathematics Subject Classification. Primary 60F15; Secondary 60F05, 60J60, 60B20, $60 \mathrm{H} 20,70 \mathrm{~F} 10,82 \mathrm{C} 22,33 \mathrm{C} 67$.

Key words and phrases. Interacting particle systems, Calogero-Moser-Sutherland models, zeros of Hermite polynomials, zeros of Laguerre polynomials, Hermite ensembles, Laguerre ensembles. 
The transition probabilities of the diffusions $\left(X_{t}\right)_{t \geq 0}$ on $C_{N}$ (with $C_{N}=C_{N}^{A}$ or $\left.C_{N}^{B}\right)$ are as follows by $\left[\mathrm{R}, \mathrm{RV1}, \mathrm{RV2}\right.$ : For any $x \in C_{N}$, and $E \subset C_{N}$ Borel set,

$$
K_{t}(x, E)=c_{k} \int_{E} t^{-\gamma_{k}-N / 2} \mathrm{e}^{-\left(\|x\|^{2}+\|y\|^{2}\right) /(2 t)} J_{k}\left(\frac{x}{\sqrt{t}}, \frac{y}{\sqrt{t}}\right) \cdot w_{k}(y) d y
$$

with

$$
w_{k}^{A}(x):=\prod_{i<j}\left(x_{i}-x_{j}\right)^{2 k}, \quad w_{k}^{B}(x):=\prod_{i<j}\left(x_{i}^{2}-x_{j}^{2}\right)^{2 k_{2}} \cdot \prod_{i=1}^{N} x_{i}^{2 k_{1}},
$$

and

$$
\gamma_{k}^{A}=k N(N-1) / 2, \quad \gamma_{\left(k_{1}, k_{2}\right)}^{B}=k_{2} N(N-1)+k_{1} N
$$

respectively. The weights $w_{k}$ are homogeneous of degree $2 \gamma_{k}$. Furthermore, the $c_{k}>0$ are known normalization constants, and $J_{k}$ is a multivariate Bessel function of type $A_{N-1}$ or $B_{N}$ with multiplicities $k$ or $\left(k_{1}, k_{2}\right)$ respectively; see e.g. [R]. $J_{k}$ is analytic on $\mathbb{C}^{N} \times \mathbb{C}^{N}$ with $J_{k}(x, y)>0$ for $x, y \in \mathbb{R}^{N}$. Moreover, $J_{k}(x, y)=J_{k}(y, x)$ and $J_{k}(0, y)=1$ for all $x, y \in \mathbb{C}^{N}$.

Therefore, if $X_{0}=0$, then for $t>0, X_{t}$ has the Lebesgue density

$$
\frac{c_{k}}{t^{\gamma_{k}+N / 2}} e^{-\|y\|^{2} /(2 t)} \cdot w_{k}(y) d y
$$

on $C_{N}$ for $t>0$. In particular, in the case $A_{N-1}$ for $k=1 / 2,1,2, X_{t}$ has the distribution of the eigenvalues of Gaussian orthogonal, unitary, and symplectic ensembles up to scalings; see e.g. [M]. Moreover, for general $k>0$, these distributions appear as spectral distributions of the tridiagonal $\beta$-Hermite ensembles of Dumitriu and Edelman [DE. Similar interpretations exist in the case $B_{N}$ for Laguerre ensembles and the tridiagonal $\beta$-Laguerre ensembles of [DE].

In this paper we use the generators (1.1), (1.2) and the associated stochastic differential equations for the diffusions $\left(X_{t}\right)_{t \geq 0}$ and construct polynomials $p_{l}$ in $N+1$ variables of order $l=1, \ldots, N$ such that the processes $\left(p_{l}\left(X_{t}, t\right)\right)_{t \geq 0}$ are martingales. The functions $p_{l}$ are constructed via elementary symmetric polynomials such that, up to some rescaling of the processes $\left(X_{t}\right)_{t \geq 0}$, these polynomials do not depend on the parameter $k$ in the case $A_{N-1}$, while they depend on one parameter only in the case $B_{N}$. Moreover, due to this observation, our martingale result can be also extended to the case where the independent parameter is equal to $\infty$, in which case the SDE of the renormalization of $\left(X_{t}\right)_{t \geq 0}$ simplifies to an ODE. The solution of this ODE starting in the origin $0 \in C_{N}$ can be described explicitely in terms of the zeros of the Hermite polynomial $H_{N}$ or the Laguerre polynomial $L_{N}^{(\alpha)}$ with a suitable $\alpha$ in the cases $A_{N-1}$ or $B_{N}$ respectively. This observation will lead to closed formulas for

$$
\mathbb{E}\left(\prod_{i=1}^{N}\left(y-X_{t, i}^{\beta}\right)\right) \quad(y \in \mathbb{R})
$$

for the processes $\left(X_{t}\right)_{t \geq 0}$ starting in 0 , which involve Hermite and Laguerre polynomials. These formulas are known for the multiplicities associated with the classical random matrix ensembles $[\mathrm{DG}, \mathrm{FG}$.

This paper is organized as follows: In Section 2 we consider the case $A_{N-1}$ while Section 3 is devoted to the case $B_{N}$.

We finally recapitulate the following well-known result (see Lemma 3.4, Corollary 6.6, and Proposition 6.8 of $[\mathrm{CGY}$ ] and $[\mathrm{Sch}, \mathrm{GM}$ ] for corrections of the proofs of these results) which will be the basis for our SDE approach: 
Theorem 1.1. Let $k>0$ or $k_{1}, k_{2}>0$ in the $A_{N-1}$ - or $B_{N}$-case. Then, for each starting point $x \in C_{N}$ and $t>0$, the initial problem

$$
X_{0}=x, \quad d X_{t}=d B_{t}+\frac{1}{2}\left(\nabla\left(\ln w_{k}\right)\right)\left(X_{t}\right) d t
$$

where $w_{k}$ is defined by (1.4) and $\left(B_{t}\right)_{t \geq 0}$ is an $N$-dimensional Brownian motion, has a unique strong solution $\left(X_{t}\right)_{t \geq 0}$. This solution is a Bessel process as above.

Moreover, if $k \geq 1 / 2$ in the $A_{N-1}$-case or $k_{1}, k_{2} \geq 1 / 2$ in the $B_{N}$-case, then $X_{t}$ is in the interior on $C_{N}$ almost surely for $t>0$.

\section{Bessel processes of type A}

We now study Bessel processes of type $A_{N-1}$ where we denote the multiplicities by $\beta \geq 0$ instead of $k$ in order to avoid confusions with indices and coordinates. Therefore, let $\left(X_{t}^{\beta}\right)_{t \geq 0}$ be a Bessel process of type $A_{N-1}$ with multiplicity $\beta \geq 0$ with values from

$$
C_{N}^{A}:=\left\{x \in \mathbb{R}^{N}: \quad x_{1} \geq x_{2} \geq \ldots \geq x_{N}\right\} .
$$

$\left(X_{t}^{\beta}\right)_{t \geq 0}$ satisfies the SDE

$$
d X_{t, i}^{\beta}=d B_{t, i}+\beta \sum_{j: j \neq i} \frac{1}{X_{t, i}^{\beta}-X_{t, j}^{\beta}} d t \quad(i=1, \ldots, N) .
$$

with an $N$-dimensional Brownian motion $\left(B_{t, 1}, \ldots, B_{t, N}\right)_{t \geq 0}$, where the paths are reflected whenever they hit the boundary $\partial C_{N}^{A}$ of $C_{N}^{A}$. By Theorem 1.1. $\left(X_{t}^{\beta}\right)_{t \geq 0}$ does not meet $\partial C_{N}^{A}$ for $t>0$ a.s. for $\beta \geq 1 / 2$.

For the sake of convenience we will study the renormalized processes $\left(\widetilde{X}_{t}:=\right.$ $\left.\widetilde{X}_{t}^{\beta}:=X_{t}^{\beta} / \sqrt{\beta}\right)_{t \geq 0}$ which satisfy

$$
d \widetilde{X}_{t, i}^{\beta}=\frac{1}{\sqrt{\beta}} d B_{t, i}+\sum_{j: j \neq i} \frac{1}{\widetilde{X}_{t, i}^{\beta}-\widetilde{X}_{t, j}^{\beta}} d t \quad(i=1, \ldots, m) .
$$

¿From now on the parameter $\beta$ in $\widetilde{X}_{t}^{\beta}$ will be omitted unless it is explicitly referred to.

Now we will derive some results for symmetric polynomials of $\left(X_{t}\right)_{t>0}$ and $\left(\widetilde{X}_{t}\right)_{t \geq 0}$. First let us we recapitulate that the elementary symmetric polynomials $e_{k}^{m}(m \in \mathbb{N}, k=0, \ldots, m)$ in $m$ variables are characterized by

$$
\prod_{k=1}^{m}\left(z-x_{k}\right)=\sum_{k=0}^{m}(-1)^{m-k} e_{m-k}^{m}(x) z^{k} \quad\left(z \in \mathbb{C}, x=\left(x_{1}, \ldots, x_{m}\right)\right),
$$

in particular, $e_{0}^{m}=1, e_{1}^{m}(x)=\sum_{k=1}^{m} x_{k}, \quad \ldots \quad e_{m}^{m}(x)=\prod_{k=1}^{m} x_{k}$.

We need a further notation: For a non-empty set $S \subset\{1, \ldots, N\}$, let $\widetilde{X}_{t, S}$ be the $\mathbb{R}^{|S|}$-valued random vector with the coordinates $\widetilde{X}_{t, i}$ for $i \in S$ in the natural ordering on $S$. The following technical observation is our starting point: 
Lemma 2.1. For $\beta \geq 1 / 2, k=2, \ldots, N$, and $l \geq 0$

$$
\begin{aligned}
d\left(t^{l} \cdot e_{k}^{N}\left(\widetilde{X}_{t}\right)\right)= & \frac{t^{l}}{\sqrt{\beta}} \sum_{j=1}^{N} e_{k-1}^{N-1}\left(\widetilde{X}_{t,\{1, \ldots, N\} \backslash\{j\}}\right) d B_{t, j} \\
& +\left(l t^{l-1} \cdot e_{k}^{N}\left(\widetilde{X}_{t}\right)-\frac{t^{l}}{2}(N-k+2)(N-k+1) e_{k-2}^{N}\left(\widetilde{X}_{t}\right)\right) d t .
\end{aligned}
$$

Proof. Itô's formula and the SDE (2.2) show that

$$
d\left(t^{l} \cdot e_{k}^{N}\left(\widetilde{X}_{t}\right)\right)=l t^{l-1} \cdot e_{k}^{N}\left(\widetilde{X}_{t}\right) d t+t^{l} \sum_{j=1}^{N} e_{k-1}^{N-1}\left(\widetilde{X}_{t,\{1, \ldots, N\} \backslash\{j\}}\right) d \widetilde{X}_{t, j} .
$$

Therefore, by the SDE (2.2),

$$
\begin{aligned}
d\left(t^{l} \cdot e_{k}^{N}\left(\widetilde{X}_{t}\right)\right)= & l t^{l-1} \cdot e_{k}^{N}\left(\widetilde{X}_{t}\right) d t+\frac{t^{l}}{\sqrt{\beta}} \sum_{j=1}^{N} e_{k-1}^{N-1}\left(\widetilde{X}_{t,\{1, \ldots, N\} \backslash\{j\}}\right) d B_{t, j} \\
& +t^{l} \sum_{j=1}^{N} \sum_{i: i \neq j} \frac{e_{k-1}^{N-1}\left(\widetilde{X}_{t,\{1, \ldots, N\} \backslash\{j\}}\right)}{\widetilde{X}_{t, j}-\widetilde{X}_{t, i}} d t \\
= & l t^{l-1} \cdot e_{k}^{N}\left(\widetilde{X}_{t}\right) d t+\frac{t^{l}}{\sqrt{\beta}} \sum_{j=1}^{N} e_{k-1}^{N-1}\left(\widetilde{X}_{t\{1, \ldots, N\} \backslash\{j\}}\right) d B_{t, j} \\
& +\frac{t^{l}}{2} \sum_{i, j=1, \ldots, n ; i \neq j} \frac{e_{k-1}^{N-1}\left(\widetilde{X}_{t,\{1, \ldots, N\} \backslash\{j\}}\right)-e_{k-1}^{N-1}\left(\widetilde{X}_{t,\{1, \ldots, N\} \backslash\{i\}}\right)}{\widetilde{X}_{t, j}-\widetilde{X}_{t, i}} d t .
\end{aligned}
$$

Moreover, by simple combinatorial computations (see (2.10), (2.11) in [VW]) we have for $i \neq j$ that

$e_{k-1}^{N-1}\left(\widetilde{X}_{t,\{1, \ldots, N\} \backslash\{j\}}\right)-e_{k-1}^{N-1}\left(\widetilde{X}_{t,\{1, \ldots, N\} \backslash\{i\}}\right)=\left(\widetilde{X}_{t, i}-\widetilde{X}_{t, j}\right) e_{k-2}^{N-2}\left(\widetilde{X}_{t,\{1, \ldots, N\} \backslash\{i, j\}}\right)$

and

$$
\sum_{i, j=1, \ldots, N ; i \neq j} e_{k-2}^{N-2}\left(\widetilde{X}_{t,\{1, \ldots, N\} \backslash\{i, j\}}\right)=(N-k+2)(N-k+1) e_{k-2}^{N}\left(\widetilde{X}_{t}\right) .
$$

(2.4)-(2.6) now lead to the lemma.

We also need the following well known observation. Here we always use the canonical filtration of the Brownian motion $\left(B_{t}\right)_{t \geq 0}$.

Lemma 2.2. For each polynomial $p$ in $N$ variables, $\beta \geq 0, i=1, \ldots, N$, and $l \geq 0$, the process $\left(\int_{0}^{t} s^{l} \cdot p\left(\widetilde{X}_{s}\right) d B_{s, i}\right)_{t \geq 0}$ is a martingale.

Proof. The process $\left(\sum_{i=1}^{N} X_{t, i}^{2}\right)_{t \geq 0}$ is a classical one-dimensional squared Bessel process (see e.g. [RV1]), i.e., all powers of this process are square-integrable w.r.t. the measure $\left.\mathcal{P} \otimes \lambda\right|_{[0, t]}$ on $\Omega \times[0, t]$ for each $t>0$, the probability measure $\mathcal{P}$ on the underlying probability space $\Omega$, and the Lebesgue measure $\lambda$. The lemma is now clear by the very construction of the Itô integral.

Using Lemmas 2.1 and 2.2. the following martingales can be constructed for Bessel processes via elementary symmetric polynomials: 
Proposition 2.3. For all $\beta>0, k=1, \ldots, N$, and all starting points $x_{0} \in C_{N}^{A}$ of $\left(\widetilde{X}_{t, \beta}\right)_{t \geq 0}$, the process

$$
\left(e_{k}^{N}\left(\widetilde{X}_{t}\right)+\sum_{l=1}^{\lfloor k / 2\rfloor} \frac{(N-k+2 l) !}{2^{l} \cdot l ! \cdot(N-k) !} t^{l} \cdot e_{k-2 l}^{N}\left(\widetilde{X}_{t}\right)\right)_{t \geq 0}
$$

is a martingale.

Proof. First assume $\beta \geq 1 / 2$. For $k=1$ we obtain from the SDE (2.2) that

$$
e_{1}^{N}\left(\widetilde{X}_{s}\right)=\sum_{i=1}^{N} \widetilde{X}_{s, i}=\frac{1}{\sqrt{\beta}} \sum_{i=1}^{N} B_{s, i}+e_{1}^{N}\left(x_{0}\right)
$$

which proves the proposition for $k=1$. In general, it follows from Lemmas 2.1 and 2.2 that

$$
\begin{aligned}
\left(e_{k}^{N}\left(\widetilde{X}_{t}\right)+\right. & \sum_{l=1}^{\lfloor k / 2\rfloor-1} \frac{(N-k+2 l) !}{2^{l} \cdot l ! \cdot(N-k) !} t^{l} \cdot e_{k-2 l}^{N}\left(\widetilde{X}_{t}\right) \\
& \left.+\frac{(N-k+2\lfloor k / 2\rfloor) !}{2^{\lfloor k / 2\rfloor} \cdot(\lfloor k / 2\rfloor-1) ! \cdot(N-k) !} \int_{0}^{t} s^{\lfloor k / 2\rfloor-1} \cdot e_{k-2\lfloor k / 2\rfloor}^{N}\left(\widetilde{X}_{s}\right) d s\right)_{t \geq 0}
\end{aligned}
$$

is a martingale. We thus only have to compare the last term on the RHS of (2.9) with the summand $l=\lfloor k / 2\rfloor$ on the RHS of (2.7). Here the arguments are different for even and odd $k$.

If $k$ is even, then $e_{k-2\lfloor k / 2\rfloor}^{N}=e_{0}^{N}=1$ and $\int_{0}^{t} s^{\lfloor k / 2\rfloor-1} d s=\frac{1}{\lfloor k / 2\rfloor} t^{\lfloor k / 2\rfloor}$. This shows that the last term on the RHS of (2.9) is the last summand for $l=\lfloor k / 2\rfloor$ in (2.7). This yields the claim in the even case.

Now let $k \geq 3$ be odd. Here $e_{k-2\lfloor k / 2\rfloor}^{N}=e_{1}^{N}$, and we obtain from the SDE (2.2) and Itô's formula that

$$
\begin{aligned}
t^{\lfloor k / 2\rfloor} e_{k-2\lfloor k / 2\rfloor}^{N}\left(\widetilde{X}_{t}\right)=t^{\lfloor k / 2\rfloor} \sum_{i=1}^{N} \widetilde{X}_{t, i}=\frac{t^{\lfloor k / 2\rfloor}}{\sqrt{\beta}} \sum_{i=1}^{N} B_{t, i}+t^{\lfloor k / 2\rfloor} e_{1}^{N}\left(x_{0}\right) \\
=\frac{1}{\sqrt{\beta}} \int_{0}^{t} s^{\lfloor k / 2\rfloor} d\left(\sum_{i=1}^{N} B_{s, i}\right)+\frac{\lfloor k / 2\rfloor}{\sqrt{\beta}} \int_{0}^{t}\left(\sum_{i=1}^{N} B_{s, i}\right) s^{\lfloor k / 2\rfloor-1} d s+t^{\lfloor k / 2\rfloor} e_{1}^{N}\left(x_{0}\right) \\
=\frac{1}{\sqrt{\beta}} \int_{0}^{t} s^{\lfloor k / 2\rfloor} d\left(\sum_{i=1}^{N} B_{s, i}\right) \\
\quad+\lfloor k / 2\rfloor \int_{0}^{t}\left(e_{1}^{N}\left(\widetilde{X}_{s}\right)-e_{1}^{N}\left(x_{0}\right)\right) s^{\lfloor k / 2\rfloor-1} d s+t^{\lfloor k / 2\rfloor} e_{1}^{N}\left(x_{0}\right) \\
=\frac{1}{\sqrt{\beta}} \int_{0}^{t} s^{\lfloor k / 2\rfloor} d\left(\sum_{i=1}^{N} B_{s, i}\right)+\lfloor k / 2\rfloor \int_{0}^{t} e_{1}^{N}\left(\widetilde{X}_{s}\right) s^{\lfloor k / 2\rfloor-1} d s .
\end{aligned}
$$

This and (2.9) yield the proposition for $k \geq 3$ odd. In summary, the proposition holds for $\beta \geq 1 / 2$.

We now use Dynkin's formula (see e.g. Section III.10 of [RW]) which implies that the symmetric functions

$$
f_{N, k}: C_{N}^{A} \times\left[0, \infty\left[\rightarrow \mathbb{R}, \quad(x, t) \mapsto e_{k}^{N}(x)+\sum_{l=1}^{\lfloor k / 2\rfloor} \frac{(N-k+2 l) !}{2^{l} \cdot l ! \cdot(N-k) !} t^{l} \cdot e_{k-2 l}^{N}(x)\right.\right.
$$


are space-time-harmonic w.r.t. the generators

$$
\widetilde{\Delta}_{\beta} f=\frac{1}{2 \sqrt{\beta}} \Delta f+\sum_{i=1}^{N}\left(\sum_{j \neq i} \frac{1}{x_{i}-x_{j}}\right) \frac{\partial}{\partial x_{i}} f
$$

of the diffusions $\left(\widetilde{X}_{t}\right)_{t \geq 0}$ for $\beta \geq 1 / 2$, i.e., we have

$$
\left(\frac{\partial}{\partial t}+\widetilde{\Delta}_{\beta}\right) f_{N, k} \equiv 0 .
$$

As the left hand side of (2.10) is analytic in $\beta$, analytic continuation shows that $f_{N, k}$ is space-time-harmonic also for all $\beta>0$. Dynkin's formula now yields the proposition in general.

Notice that the functions $f_{N, k}$ do not depend on $\beta>0$, and that the simultanous space-time harmonicity w.r.t. all $\widetilde{\Delta}_{\beta}(\beta>0)$ is trivial, as $\beta$ only appears as a factor of the classical Laplacian $\Delta$, for which obviously $\Delta e_{k}^{N} \equiv$ holds for all $k$.

We also point out that Lemma 2.1 and Proposition 2.3 remain valid for $\beta=\infty$, in which case the SDE (2.2) is an ODE, and the process $\left(\widetilde{X}_{t}^{\infty}\right)_{t>0}$ is deterministic whenever so is the initial condition for $t=0$. There are several limit theorems (laws of large numbers, CLTs) for the limit transition $\beta \rightarrow \infty$; see [AKM1, AV] [VW]. Proposition $[2.3$ for $\beta \in] 0, \infty]$ leads to:

Corollary 2.4. Let $\left(\widetilde{X}_{t}^{\beta}\right)_{t \geq 0}$ be a normalized Bessel process for $\left.\left.\beta \in\right] 0, \infty\right]$ which starts in some $x_{0} \in C_{N}^{A}$. Then for $k=0,1, \ldots, N$ and $t \geq 0$, the expectations $\mathbb{E}\left(e_{k}^{N}\left(\widetilde{X}_{t}^{\beta}\right)\right)$ do not depend on $\beta$.

Proof. The the case $k=0$ is trivial, and, by (2.8), $\mathbb{E}\left(e_{1}^{N}\left(\tilde{X}_{t}^{\beta}\right)\right)=0$, which proves the result for $k=1$. Proposition 2.3 and induction now lead to the general case.

We now study the case when the process is initially in the origin. For $\beta=\infty$, the solution of the ODE (2.2) can be expressed via the ordered zeros $z_{1}>z_{2}>\ldots>z_{N}$ of the Hermite polynomial

$$
H_{N}(x)=\sum_{k=0}^{\lfloor k / 2\rfloor}(-1)^{k} \frac{N !}{k !(N-2 k) !} 2^{N-2 k} x^{N-2 k}
$$

where the Hermite polynomials $\left(H_{N}\right)_{N \geq 0}$ are orthogonal w.r.t. the density $e^{-x^{2}}$; see [S] for details. We have the following result by [AV] which follows easily from Section 6.7 of $[\mathrm{S}]$ on the zeros of $H_{N}$ :

Lemma 2.5. The solution of the $O D E$ (2.2) with $\beta=\infty$ and start in $0 \in C_{N}^{A}$ is given by $\widetilde{X}_{t}^{\infty}=\sqrt{2 t} \cdot z$ with $z:=\left(z_{1}, \ldots, z_{N}\right)$.

This and the preceding results have the following consequence:

Corollary 2.6. For $\beta \in] 0, \infty\left[\right.$ let $\left(X_{t, \beta}\right)_{t \geq 0}$ be the Bessel process of type $A$ with start in 0 . Then,

$$
\begin{aligned}
\mathbb{E}\left(\prod_{i=1}^{N}\left(y-X_{t, i}^{\beta}\right)\right) & =c_{\beta}^{A} \int_{\mathbb{R}^{N}}\left(\prod_{i=1}^{N}\left(y-x_{i}\right)\right) \cdot t^{-\gamma_{\beta}^{A}-N / 2} \mathrm{e}^{-\|x\|^{2} /(2 t)} \prod_{i<j}\left(x_{i}-x_{j}\right)^{2 \beta} d x \\
& =(t \beta / 2)^{N / 2} \cdot H_{N}(y / \sqrt{2 \beta t}) \quad \text { for } \quad y \in \mathbb{R} .
\end{aligned}
$$


Moreover, for $k=0,1, \ldots,\lfloor k / 2\rfloor-1, \mathbb{E}\left(e_{2 k+1}^{N}\left(X_{t}^{\beta}\right)\right)=0$, and

$$
\mathbb{E}\left(e_{2 k}^{N}\left(X_{t}^{\beta}\right)\right)=(t \beta / 2)^{k} \frac{N !}{k !(N-2 k) !} \quad(k=0,1, \ldots,\lfloor k / 2\rfloor) .
$$

Proof. Corollary 2.4 and Lemma 2.5 yield

$$
\begin{aligned}
& \mathbb{E}\left(\prod_{i=1}^{N}\left(y-X_{t, i}^{\beta}\right)\right)=\sum_{k=0}^{N}(-1)^{k} \mathbb{E}\left(e_{k}^{N}\left(X_{t}^{\beta}\right)\right) \cdot y^{N-k} \\
& =\beta^{N / 2} \sum_{k=0}^{N}(-1)^{k} \mathbb{E}\left(e_{k}^{N}\left(\tilde{X}_{t}^{\beta}\right)\right)(y / \sqrt{\beta})^{N-k} \\
& =\beta^{N / 2} \sum_{k=0}^{N}(-1)^{k} \mathbb{E}\left(e_{k}^{N}\left(\tilde{X}_{t}^{\infty}\right)\right)(y / \sqrt{\beta})^{N-k} \\
& =\beta^{N / 2} \sum_{k=0}^{N}(-1)^{k} e_{k}^{N}(\sqrt{2 t} \cdot z)(y / \sqrt{\beta})^{N-k} \\
& =(2 t \beta)^{N / 2} \sum_{k=0}^{N}(-1)^{k} e_{k}^{N}(z)(y / \sqrt{2 t \beta})^{N-k} \\
& =(2 t \beta)^{N / 2} \prod_{i=1}^{N}\left(y / \sqrt{2 t \beta}-z_{i}\right)=(2 t \beta)^{N / 2} \frac{1}{2^{N}} \cdot H_{N}(y / \sqrt{2 t \beta}) .
\end{aligned}
$$

This proves the first statement. The second one follows by a comparison of the coefficients in (2.11) and (2.14).

Remark 2.7. For $\beta=1 / 2,1,2$ and start in 0 , the random variables $X_{t}^{\beta}$ have the same distributions as the ordered eigenvalues of a Gaussian orthogonal, unitary, or symplectic ensemble processes respectively up to normalizations by (1.6). In this way, Corollary 2.6 can be restated for these ensembles. In particular, (2.12) yields Proposition 11 of [FG] in the Gaussian unitary case; see also [DG].

Remark 2.8. By $\mathrm{AV}$, the solution of the ODE (2.2) with $\beta=\infty$ and start in $c z$, $c \geq 0$, is given by $\sqrt{2 t+c^{2}} z$. For Bessel processes of type A with start in $c z$, this leads to

$$
\mathbb{E}\left(\prod_{i=1}^{N}\left(y-X_{t, i}^{\beta}\right)\right)=\left(\left(2 t+c^{2}\right) \beta / 4\right)^{N / 2} \cdot H_{N}\left(y / \sqrt{\left(2 t+c^{2}\right) \beta}\right) .
$$

Remark 2.9. All preceding results are concerned with formulas which are invariant under the canonical action of the symmetric group $S_{N}$ on $\mathbb{R}^{N}$. We thus can replace the Bessel processes $\left(X_{t}^{\beta}\right)_{t \geq 0}$ by Dunkl processes of type $A_{N-1}$ in Proposition 2.3 and Corollaries 2.4 and 2.6. For the theory of Dunkl processes we refer to CGY, GY, RV1, RV2.

Remark 2.10. Corollary 2.6 is also valid for the case $\beta=0$. Here, the Dunkl process is an $N$-dimensional Brownian motion, and the Bessel process a Brownian motion on $C_{N}^{A}$ which is reflected on $\partial C_{N}^{A}$. We here have $\mathbb{E}\left(e_{k}^{N}\left(X_{t}^{0}\right)\right)=0$ for $k \geq 1$. 


\section{Bessel PROCEsses of type B}

In this section we study Bessel processes for the root systems $B_{N}$ with multiplicities $\left(k_{1}, k_{2}\right):=(\nu \cdot \beta, \beta)$ with parameters $\nu \geq 0, \beta>0$. These processes $\left(X_{t}:=\left(X_{t, 1}, \ldots, X_{t, N}\right)\right)_{t \geq 0}$ have values on the closed Weyl chamber

$$
C_{N}^{B}:=\left\{x \in \mathbb{R}^{N}: \quad x_{1} \geq x_{2} \geq \ldots \geq x_{N} \geq 0\right\}
$$

of type B and satisfy the SDE

$$
d X_{t, i}^{\beta}=d B_{t, i}+\beta \sum_{j \neq i}\left(\frac{1}{X_{t, i}^{\beta}-X_{t, j}^{\beta}}+\frac{1}{X_{t, i}^{\beta}+X_{t, j}^{\beta}}\right) d t+\frac{\nu \cdot \beta}{X_{t, i}^{\beta}} d t
$$

for $i=1, \ldots, N$ with an $N$-dimensional Brownian motion $\left(B_{t}\right)_{t \geq 0}$ where the paths are reflected when they meet the boundary $\partial C_{N}^{B}$ of $C_{N}^{B}$. Again, by Proposition 6.1 of [CGY], the process does not meet the boundary in positive time almost surely for $\beta \geq 1 / 2$ and $\nu \geq 1$.

Again we also study the renormalized processes $\left(\widetilde{X}_{t}^{\beta}:=X_{t} / \sqrt{\beta}\right)_{t \geq 0}$ with

$$
d \widetilde{X}_{t, i}^{\beta}=\frac{1}{\sqrt{\beta}} d B_{t, i}+\sum_{j \neq i}\left(\frac{1}{\widetilde{X}_{t, i}^{\beta}-\widetilde{X}_{t, j}^{\beta}}+\frac{1}{\widetilde{X}_{t, i}^{\beta}+\widetilde{X}_{t, j}^{\beta}}\right) d t+\frac{\nu}{\widetilde{X}_{t, i}^{\beta}} d t
$$

for $i=1, \ldots, N$ where we usually omit the parameter $\beta$ in $\tilde{X}_{t}^{\beta}$. We now derive an analogue of Lemma 2.1 which involves functions, which are invariant under the Weyl group of type $B_{N}$. As in the proof of Lemma 2.1] let $\widetilde{X}_{t, S}$ be the $\mathbb{R}^{|S|}$-valued random vector with the coordinates $\widetilde{X}_{t, i}$ for $i \in S$ in the natural ordering on a subset $S \subset\{1, \ldots, N\}$.

Lemma 3.1. For all $\beta \geq 1 / 2, \nu \geq 1, k=1,2, \ldots, N$, and $l \geq 0$

$$
\begin{aligned}
d\left(t^{l} \cdot e_{k}^{N}\left(\widetilde{X}_{t}^{2}\right)\right) & =\frac{2 t^{l}}{\sqrt{\beta}} \sum_{j=1}^{N} \widetilde{X}_{t, j} \cdot e_{k-1}^{N-1}\left(\widetilde{X}_{t,\{1, \ldots, N\} \backslash\{j\}}^{2}\right) d B_{t, j} \\
& +\left(l t^{l-1} \cdot e_{k}^{N}\left(\widetilde{X}_{t}^{2}\right)+2 t^{l}(N-k+\nu+1 /(2 \beta))(N-k+1) e_{k-1}^{N}\left(\widetilde{X}_{t}^{2}\right)\right) d t .
\end{aligned}
$$

Proof. As $d\left[\widetilde{X}_{t, i}, \widetilde{X}_{t, j}\right]=\frac{\delta_{i, j}}{\beta} d t$ by (3.2), Itô's formula implies that

$$
\begin{aligned}
d\left(t^{l} \cdot e_{k}^{N}\left(\widetilde{X}_{t}^{2}\right)\right)= & l t^{l-1} \cdot e_{k}^{N}\left(\widetilde{X}_{t}^{2}\right) d t+t^{l} \sum_{j=1}^{N} 2 \widetilde{X}_{t, j} \cdot e_{k-1}^{N-1}\left(\widetilde{X}_{t,\{1, \ldots, N\} \backslash\{j\}}^{2}\right) d \widetilde{X}_{t, j} \\
& +\frac{t^{l}}{\beta} \sum_{j=1}^{N} e_{k-1}^{N-1}\left(\widetilde{X}_{t,\{1, \ldots, N\} \backslash\{j\}}^{2}\right) d t .
\end{aligned}
$$


Therefore, by the SDE (3.2),

$$
\begin{aligned}
d\left(t^{l} \cdot e_{k}^{N}\left(\widetilde{X}_{t}^{2}\right)\right) & =l t^{l-1} \cdot e_{k}^{N}\left(\widetilde{X}_{t}^{2}\right) d t+\frac{2 t^{l}}{\sqrt{\beta}} \sum_{j=1}^{N} \widetilde{X}_{t, j} \cdot e_{k-1}^{N-1}\left(\widetilde{X}_{t,\{1, \ldots, N\} \backslash\{j\}}^{2}\right) d B_{t, j} \\
& +2 t^{l} \sum_{i, j ; i \neq j}\left(\frac{e_{k-1}^{N-1}\left(\widetilde{X}_{t,\{1, \ldots, N\} \backslash\{j\}}^{2}\right) X_{t, j}}{\widetilde{X}_{t, j}-\widetilde{X}_{t, i}}+\frac{e_{k-1}^{N-1}\left(\widetilde{X}_{t,\{1, \ldots, N\} \backslash\{j\}}^{2}\right) X_{t, j}}{\widetilde{X}_{t, j}+\widetilde{X}_{t, i}}\right) d t \\
& +2 t^{l} \nu \sum_{j=1}^{N} e_{k-1}^{N-1}\left(\widetilde{X}_{t,\{1, \ldots, N\} \backslash\{j\}}^{2}\right) d t+\frac{t^{l}}{\beta} \sum_{j=1}^{N} e_{k-1}^{N-1}\left(\widetilde{X}_{t,\{1, \ldots, N\} \backslash\{j\}}^{2}\right) d t \\
& =l t^{l-1} \cdot e_{k}^{N}\left(\widetilde{X}_{t}^{2}\right) d t+\frac{2 t^{l}}{\sqrt{\beta}} \sum_{j=1}^{N} \widetilde{X}_{t, j} \cdot e_{k-1}^{N-1}\left(\widetilde{X}_{t,\{1, \ldots, N\} \backslash\{j\}}^{2}\right) d B_{t, j} \\
& +2 t^{l} \sum_{i, j ; i \neq j}\left(\frac{\widetilde{X}_{t, j}^{2} e_{k-1}^{N-1}\left(\widetilde{X}_{t,\{1, \ldots, N\} \backslash\{j\}}^{2}\right)-\widetilde{X}_{t, i}^{2} e_{k-1}^{N-1}\left(\widetilde{X}_{t,\{1, \ldots, N\} \backslash\{i\}}^{2}\right)}{\widetilde{X}_{t, j}^{2}-\widetilde{X}_{t, i}^{2}}\right) d t \\
& +2 t^{l}(\nu+1 /(2 \beta)) \sum_{j=1}^{N} e_{k-1}^{N-1}\left(\widetilde{X}_{t,\{1, \ldots, N\} \backslash\{j\}}^{2}\right) d t .
\end{aligned}
$$

Simple combinatorial computations (cf. (4.9)-(4.12) in [VW] show for $i \neq j$ and $k \geq 2$ that

$$
\begin{gathered}
\tilde{X}_{t, j}^{2} e_{k-1}^{N-1}\left(\widetilde{X}_{t,\{1, \ldots, N\} \backslash\{j\}}^{2}\right)-\widetilde{X}_{t, i}^{2} e_{k-1}^{N-1}\left(\widetilde{X}_{t,\{1, \ldots, N\} \backslash\{i\}}^{2}\right) \\
=\left(\widetilde{X}_{t, j}^{2}-\widetilde{X}_{t, i}^{2}\right) \cdot e_{k-1}^{N-2}\left(\widetilde{X}_{t,\{1, \ldots, N\} \backslash\{i, j\}}^{2}\right), \\
\sum_{i, j=1, \ldots, N ; i \neq j} e_{k-1}^{N-2}\left(\widetilde{X}_{t,\{1, \ldots, N\} \backslash\{i, j\}}^{2}\right)=(N-k+1)(N-k) e_{k-1}^{N}\left(\widetilde{X}_{t}^{2}\right),
\end{gathered}
$$

and

$$
\sum_{j=1}^{N} e_{k-1}^{N-1}\left(\widetilde{X}_{t,\{1, \ldots, N\} \backslash\{j\}}^{2}\right)=(N-k+1) e_{k-1}^{N}\left(\widetilde{X}_{t}^{2}\right) .
$$

(3.4)-(3.6), and (3.3) now lead to the lemma for $k \geq 2$. For $k=1$, the lemma also follows by an even simpler computation.

We also have the following analogue of Lemma 2.2 by the same reasons. The proof is very similar, hence omitted.

Lemma 3.2. For each polynomial $p$ in $N$ variables, $i=1, \ldots, N$, and $l \geq 0$, the process $\left(\int_{0}^{t} s^{l} \cdot p\left(\widetilde{X}_{s}\right) d B_{s}^{i}\right)_{t \geq 0}$ is a martingale.

With Lemmas 3.1 and 3.2. we obtain the following martingales:

Proposition 3.3. For all $\nu \geq 0, \beta>0, k=1, \ldots, N$, and all starting points $\widetilde{X}_{0, \beta} \in C_{N}^{B}$, the process

$$
\left(e_{k}^{N}\left(\widetilde{X}_{t}^{2}\right)+\sum_{l=1}^{k}(-2 t)^{l}\left(\begin{array}{c}
N-k+l \\
l
\end{array}\right)(N-k+\nu+1 /(2 \beta))_{l} \cdot e_{k-l}^{N}\left(\widetilde{X}_{t}^{2}\right)\right)_{t \geq 0}
$$

(with the Pochhammer symbol $(x)_{r}:=x(x+1) \cdots(x+r-1)$ ) is a martingale. 
Proof. For $\beta \geq 1 / 2$ and $\nu \geq 1$, it follows readily from Lemmas 3.1 and 3.2 that

$$
\begin{aligned}
\left(e_{k}^{N}\left(\widetilde{X}_{t}^{2}\right)+\right. & \sum_{l=1}^{k-1}(-2 t)^{l}\left(\begin{array}{c}
N-k+l \\
l
\end{array}\right)(N-k+\nu+1 /(2 \beta))_{l} e_{k-l}^{N}\left(\widetilde{X}_{t}^{2}\right) \\
& \left.+(-2)^{k} \cdot\left(\begin{array}{c}
N \\
k
\end{array}\right) k(N-k+\nu+1 /(2 \beta))_{k} \int_{0}^{t} s^{k-1} d s\right)_{t \geq 0}
\end{aligned}
$$

is a martingale which proves the claim in this case. The extension to arbitrary $\beta, \nu$ follows again by "analytic continuation" as in the proof of Proposition 2.3

Notice that the algebraic functions in Proposition 3.3. which lead to martingales, depend on $\nu+1 /(2 \beta)$ only. Moreover, Lemma 3.1 and Proposition 3.3 remain valid for $\beta=\infty$, in which case the SDE (3.2) is an ODE, and the process $\left(\widetilde{X}_{t}\right)_{t \geq 0}$ is deterministic whenever so is the initial condition for $t=0$. There are several limit theorems (laws of large numbers, CLTs) for the limit transition $\beta \rightarrow \infty$; see AKM2, [AV], VW]. Proposition 3.3 for $\beta \in] 0, \infty]$ leads to:

Corollary 3.4. For a fixed $x_{0} \in C_{N}^{A}$, let $\left(\widetilde{X}_{t}\right)_{t \geq 0}$ be a Bessel process of type $B$ with start in $x_{0}$ for the parameters $\nu \geq 0$ and $\left.\left.\beta \in\right] 0, \infty\right]$. Then for $k=0,1, \ldots, N$ and $t \geq 0$, the expectations $\mathbb{E}\left(e_{k}^{N}\left(\widetilde{X}_{t}^{2}\right)\right)$ depend on $\nu+1 /(2 \beta)$ only (and not on $\nu, \beta$ ).

Proof. This is clear by Proposition 3.3 and induction.

We now start in $x_{0}=0 \in C_{N}^{A}$. Then for $\beta=\infty$, the solution of the ODE (3.2) can be written via zeros of some Laguerre polynomial. For this we recapitulate that for $\alpha>0$, the Laguerre polynomials

$$
L_{n}^{(\alpha)}(x)=\sum_{k=0}^{n}\left(\begin{array}{l}
n+\alpha \\
n-k
\end{array}\right) \frac{(-x)^{k}}{k !}
$$

are orthogonal w.r.t. the density $e^{-x} \cdot x^{\alpha}$ on $] 0, \infty[$ as in $[\underline{\mathrm{S}}$. We need the following fact; see [AKM1, Section 6.7 of [S], or, in the present notation, $\underline{\mathrm{AV}}$ ]:

Lemma 3.5. Let $\nu>0$ and denote by $z_{1}^{(\nu-1)}>\ldots>z_{N}^{(\nu-1)}>0$ the ordered zeros of $L_{N}^{(\nu-1)}$. Then the vector $y \in C_{N}^{B}$ with $y^{2}:=\left(y_{1}^{2}, \ldots, y_{N}^{2}\right)=2\left(z_{1}^{(\nu-1)}, \ldots, z_{N}^{(\nu-1)}\right)$ satisfies

$$
\frac{1}{2} y_{i}=\sum_{j: j \neq i}\left(\frac{1}{y_{i}-y_{j}}+\frac{1}{y_{i}+y_{j}}\right)+\frac{\nu}{y_{i}} \quad(i=1, \ldots, N)
$$

This leads to the following solutions of the ODEs (3.2) for $\beta=\infty$; cf. [AV]:

Corollary 3.6. Let $\nu>0$ and $y \in C_{N}^{B}$ the vector in Lemma 3.5. Then $\phi(t)=\sqrt{t} \cdot y$ is a solution of (3.2) for $\beta=\infty$.

This result has the following consequence: 
Corollary 3.7. Let $\left(X_{t}\right)_{t \geq 0}$ be the Bessel process of type $B$ starting in 0 with parameters $\nu \geq 0, \beta>0$. Then,

$$
\begin{aligned}
\mathbb{E}\left(\prod_{i=1}^{N}\left(y-X_{t, i}^{2}\right)\right)=c_{(\beta \nu, \beta)}^{B} \int_{\mathbb{R}^{N}} & \left(\prod_{i=1}^{N}\left(y-x_{i}^{2}\right)\right) \cdot t^{-\gamma_{(\beta \nu, \beta)}^{B}-N / 2} \mathrm{e}^{-\|x\|^{2} /(2 t)} \\
& \times \prod_{i<j}\left(x_{i}^{2}-x_{j}^{2}\right)^{2 \beta} \prod_{i=1}^{N} x_{i}^{2 \beta \nu} d x \\
= & (2 t \beta)^{N} \cdot(-1)^{N} N ! \cdot L_{N}^{(\nu+1 /(2 \beta)-1)}(y /(2 t \beta)) \quad \text { for } \quad y \in \mathbb{R} .
\end{aligned}
$$

Moreover, for $k=0,1, \ldots, N$,

$$
\mathbb{E}\left(e_{k}^{N}\left(X_{t}^{2}\right)\right)=\left(\begin{array}{c}
N+\nu+1 /(2 \beta)-1 \\
k
\end{array}\right) \cdot \frac{N !}{(N-k) !} \cdot(2 t \beta)^{k} .
$$

Proof. As here we need processes with different parameters, we denote the Bessel processes and their normalizations with parameters $\nu, \beta$ by $\left(X_{t}(\nu, \beta)\right)_{t \geq 0}$ and $\left(\widetilde{X}_{t}(\nu, \beta)\right)_{t \geq 0}$ respectively. Corollary 3.4 and Lemma 3.6 yield

$$
\begin{aligned}
\mathbb{E} & \left(\prod_{i=1}^{N}\left(y-X_{t, i}(\nu, \beta)^{2}\right)\right)=\sum_{k=0}^{N}(-1)^{k} \mathbb{E}\left(e_{k}^{N}\left(X_{t}(\nu, \beta)^{2}\right)\right) \cdot y^{N-k} \\
& =\beta^{N} \sum_{k=0}^{N}(-1)^{k} \mathbb{E}\left(e_{k}^{N}\left(\widetilde{X}_{t}(\nu, \beta)^{2}\right)\right) \cdot(y / \beta)^{N-k} \\
& =\beta^{N} \sum_{k=0}^{N}(-1)^{k} \mathbb{E}\left(e_{k}^{N}\left(\widetilde{X}_{t}(\nu+1 /(2 \beta), \infty)^{2}\right)\right) \cdot(y / \beta)^{N-k} \\
& =\beta^{N} \sum_{k=0}^{N}(-1)^{k} e_{k}^{N}\left(2 t \cdot\left(z_{1}^{(\nu+1 /(2 \beta)-1)}, \ldots, z_{N}^{(\nu+1 /(2 \beta)-1)}\right)\right) \cdot(y / \beta)^{N-k} \\
& =(2 t \beta)^{N} \sum_{k=0}^{N}(-1)^{k} e_{k}^{N}\left(z_{1}^{(\nu+1 /(2 \beta)-1)}, \ldots, z_{N}^{(\nu+1 /(2 \beta)-1)}\right) \cdot(y /(2 t \beta))^{N-k} \\
& =(2 t \beta)^{N} \prod_{i=1}^{N}\left(y /(2 t \beta)-z_{i}^{(\nu+1 /(2 \beta)-1)}\right) \\
& =(2 t \beta)^{N} \cdot(-1)^{N} N ! \cdot L_{N}^{(\nu+1 /(2 \beta)-1)}(y /(2 t \beta)) .
\end{aligned}
$$

Notice that the last equation follows from the fact that $L_{N}^{(\nu+1 /(2 \beta)-1)}$ has the leading coefficient $(-1)^{N} / N$ !; see (5.1.8) in [S]. This proves the first statement. The second statement follows by a comparison of the coefficients in (3.11) and (3.7).

Remark 3.8. Corollary 3.7 can be also applied in the limit case $\beta=0$. In this case, the Bessel process is independent of $\nu$ and a Brownian motion on $C_{N}^{B}$ where the paths are reflected on $\partial C_{N}^{B}$.

Moreover, writing $\mathbb{E}\left(e_{k}^{N}\left(X_{t}(\nu, \beta)^{2}\right)\right)$ as an integral over $C_{N}^{B}$ with the explicit densities of the random variables $X_{t}(\nu, \beta)$ for arbitrary $\beta, \nu \geq 0$ as in RV1, implies by dominated convergence that $\mathbb{E}\left(e_{k}^{N}\left(X_{t}^{2}\right)\right)$ depends continuously on $\beta, \nu$. Hence, 
by Corollary 3.7, for $\beta=0$ and $k=0,1, \ldots, N$,

$$
\mathbb{E}\left(e_{k}^{N}\left(X_{t}^{2}\right)\right)=(2 t)^{k}\left(\begin{array}{c}
N \\
k
\end{array}\right)
$$

Remark 3.9. For the cases $\beta=1 / 2,1,2$ and start in 0 , Corollary 3.7 admits an interpretation for Wishart processes and chiral ensembles. In particular we obtain Proposition 12 and Corollary 1 of [FG] in the Gaussian cases. For the details of the connection we refer to Section 3 of [RV2].

Remark 3.10. Corollary 3.7 suggests that it might be interesting to study the expectation of $\prod_{i=1}^{N}\left(y-X_{t, i}\right)$ for a Dunkl process $\left(X_{t}\right)_{t \geq 0}$ on $\mathbb{R}^{N}$ of type $B_{N}$ with arbitrary multiplicities $\left(k_{1}, k_{2}\right)$ with $k_{1}, k_{2}>0$ where the process starts in $0 \in \mathbb{R}^{N}$. For the theory of Dunkl processes and the notations we refer to CGY, GY, RV1, RV2. The result is however simple, as the distributions $P_{X_{t}}$ of the $X_{t}$ are invariant under sign changes in all coordinates separately. As thus $\mathbb{E}\left(e_{k}^{N}\left(X_{t}\right)\right)=0$ for $k=1, \ldots, N$, we get

$$
\mathbb{E}\left(\prod_{i=1}^{N}\left(y-X_{t, i}\right)\right)=\sum_{k=0}^{N} \mathbb{E}\left(e_{k}^{N}\left(X_{t}\right)\right) \cdot y^{N-k}=y^{N} \quad(y \in \mathbb{R}) .
$$

We also note that (3.12) coincides with the expectation of the characteristic polynomial of a general random square matrix with independent, centered elements.

Remark 3.11. Proposition 3.3 and Corollary 3.7 hold also for Bessel processes $\left(X_{t}^{D}\right)_{t \geq 0}$ of type $D_{N}$ which live on the closed Weyl chamber

$$
C_{N}^{D}=\left\{x \in \mathbb{R}^{N}: \quad x_{1} \geq \ldots \geq x_{N-1} \geq\left|x_{N}\right|\right\},
$$

and which depend on a one-dimensional multiplicity $\beta \geq 0$. These processes may be regarded a doubling of the processes $\left(X_{t}^{B}\right)_{t \geq 0}$ of type B w.r.t. the last coordinate with the same $\beta$ and $\nu=0$. More precisely, the squared processes $\left(\left(X_{t}^{D}\right)^{2}\right)_{t \geq 0}$ and $\left(\left(X_{t}^{B}\right)^{2}\right)_{t \geq 0}$ are equal in distribution. This shows that Proposition 3.3 and Corollary 3.7 remain valid for $\nu=0$ there.

Funding: The first author has been supported by the Deutsche Forschungsgemeinschaft (DFG) via RTG 2131 High-dimensional Phenomena in Probability Fluctuations and Discontinuity to visit Dortmund for the preparation of this paper.

\section{REFERENCES}

[AKM1] S. Andraus, M. Katori, S. Miyashita, Interacting particles on the line and Dunkl intertwining operator of type A: Application to the freezing regime. J. Phys. A: Math. Theor. 45 (2012) 395201.

[AKM2] S. Andraus, M. Katori, S. Miyashita, Two limiting regimes of interacting Bessel processes. J. Phys. A: Math. Theor. 47 (2014) 235201.

[AV] S. Andraus, M. Voit, Limit theorems for multivariate Bessel processes in the freezing regime. Stoch. Proc. Appl. , https://doi.org/10.1016/j.spa.2018.12.011, in press, arXiv:1804.03856.

[BF] T.H. Baker, P.J. Forrester, The Calogero-Sutherland model and generalized classical polynomials. Comm. Math. Phys. 188 (1997), 175-216.

[CGY] O. Chybiryakov, L. Gallardo, M. Yor, Dunkl processes and their radial parts relative to a root system. In: P. Graczyk et al. (eds.), Harmonic and stochastic analysis of Dunkl processes. Hermann, Paris 2008.

[DG] P. Diaconis, A. Gamburd, Random matrices, magic squares and matching polynomials. Electron. J. Combin. 11 (2004/06), no. 2, Research Paper 2, 26 pp.. 
[DV] J.F. van Diejen, L. Vinet, Calogero-Sutherland-Moser Models. CRM Series in Mathematical Physics, Springer, Berlin, 2000.

[DE] I. Dumitriu, A. Edelman, Matrix models for beta-ensembles. J. Math. Phys. 43 (2002), 5830-5847.

[FG] P. Forrester, A. Gamburd, Counting formulas associated with some random matrix averages. J. Combin. Theory A 113 (2006), 934-951.

[GY] L. Gallardo, M. Yor, Some remarkable properties of the Dunkl martingale. In: Seminaire de Probabilites XXXIX, pp. 337-356, dedicated to P.A. Meyer, vol. 1874, Lecture Notes in Mathematics, Springer, Berlin, 2006.

[GM] P. Graczyk, J. Malecki, Strong solutions of non-colliding particle systems. Electron. J. Probab. 19 (2014), 21 pp.

[M] M. Mehta, Random matrices (3rd ed.), Elsevier/Academic Press, Amsterdam, 2004.

[P] P.E. Protter, Stochastic Integration and Differential Equations. A New Approach. Springer, Berlin, 2003

[R] M. Rösler, Generalized Hermite polynomials and the heat equation for Dunkl operators. Comm. Math. Phys. 192 (1998), 519-542.

[RW] L.C.G. Rogers, D. Williams, Diffusions, Markov Processes and Martingales, Vol. 1 Foundations. Cambridge University Press 2000.

[RV1] M. Rösler, M. Voit, Markov processes related with Dunkl operators. Adv. Appl. Math. 21 (1998), 575-643.

[RV2] M. Rösler, M. Voit, Dunkl theory, convolution algebras, and related Markov processes. In: P. Graczyk et al. (eds.), Harmonic and stochastic analysis of Dunkl processes. Hermann, Paris 2008.

[Sch] B. Schapira, The Heckman-Opdam Markov processes. Probab. Theory Rel. Fields 138 (2007), 495-519.

[S] G. Szegö, Orthogonal Polynomials. Colloquium Publications (American Mathematical Society), Providence, 1939.

[VW] M. Voit, J. Woerner, Functional central limit theorems for multivariate Bessel processes in the freezing regime. Preprint 2019, arXiv:1901.08390

Eötvös Loránd University, Department of Probability Theory and Statistics, Pázmány Péter sétány 1/C., H-1117, Budapest, Hungary and Wigner Research Centre for Physics, Department of Quantum Optics and Quantum Information, Konkoly-Thege Miklós út 29-33., H-1121, Budapest, Hungary

E-mail address: koma@cs.elte.hu

Fakultät Mathematik, Technische Universität Dortmund, Vogelpothsweg 87, D-44221 Dortmund, Germany

E-mail address: michael.voit@math.tu-dortmund.de

Fakultät Mathematik, Technische Universität Dortmund, Vogelpothsweg 87, D-44221 Dortmund, Germany

E-mail address: jeannette.woerner@math.tu-dortmund.de 